\title{
TEORÍAS FEMINISTAS, TEORÍAS DE GÉNERO. UNA METATEORIZACIÓN
}

\author{
FEMINIST THEORIES, GENDER THEORIES. A METATHEORIZING
}

\author{
Maricela Guzmán Cáceres \\ Universidad Autónoma de Coahuila \\ mari68mx@yahoo.com.mx \\ https://orcid.org/0000-0003-1180-842X
}

Recibido/Received: 09/07/2021

Modificado/Modified: 30/09/2021

Aceptado/Accepted: 17/10/2021

\section{RESUMEN}

Este artículo revisa las principales teorías que dan cuenta del pensamiento feminista y de género desde las teorizaciones que iniciaron en la década de 1960 hasta la teoría queer del siglo XXI. La metodología utilizada es la metateorización de George Ritzer. Los resultados apuntan a que la historia del feminismo puede analizarse mediante un esquema que contempla tres fases históricas: la base material, la base teórica y la base operativa. Se concluye que el feminismo constituye un cuerpo de conocimientos que da cuenta de un crisol de respuestas a lo que es el hombre, la mujer y los géneros lábiles de los que habla la teoría queer y que es necesario el estudio y la acción que plantean los feminismos actuales para contribuir a que haya cada vez más vidas vivibles, ya que todavía se ve muy lejos la igualdad, la equidad y la justicia de género en la sociedad global.

\section{PALABRAS CLAVE:}

Teorías feministas, teorías de género, feminismo, metateorización, sociología del género.

\section{SUMARIO}

1.Introducción 2.Periodización histórica de las teorías feministas y de género 3.Metodología 4.Teorías feministas/Teorías de género 4.1 Teorías de la diferencia 4.2 Teorías de la desigualdad 4.2.1.Feminismo Liberal. 4.3 Teorías de la opresión 4.3.1 Feminismo radical 4.4 Teorías de la interseccionalidad 4.5 Feminismo posmoderno 4.6 Teoría Queer 4.7 Hacia una metateorización de las teorías feministas y de género 5.Conclusión

\begin{abstract}
This article reviews the main theories that account for feminist and gender thinking from theorizations that began in the 1960 s to queer theory of the 21 st century. The methodology used is George Ritzer's metatheorization. The results suggest that the history of feminism can be analyzed through a scheme that contemplates three historical phases: the material base, the theoretical base, and the operational base. It is concluded that feminism constitutes a body of knowledge that accounts for a melting pot of responses to what is man, woman and the labile genders of which queer theory speaks and that the study and action posed by current feminisms is necessary to contribute to more and more livable lives, since equality, equity and gender justice in global society are still far behind.
\end{abstract}

\section{KEY WORDS:}

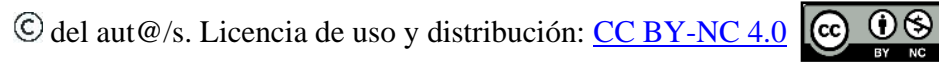


Feminist theories, gender theories, feminism, metatheorization, gender sociology.

\section{CONTENTS}

1.Introduction 2.Historical periodization of feminist and gender theories 3.Methodology 4.Feminist theories / Gender theories 4.1 Theories of difference 4.2. Theories of inequality 4.2.1.Liberal feminism 4.3.Theories of oppression 4.3.1.Radical feminism 4.4.Theories of intersectionality 4.5.Posmodern feminism 4.6.Queer theory 4.7. Towards a metatheorization of feminist and gender theories 5.Conclusion

\section{INTRODUCCIÓN}

A lo largo de los siglos, el sistema patriarcal y la situación de desventaja de las mujeres ha sido denunciado por diferentes voces, sin embargo, es a partir de los años sesenta del siglo XX, cuando la teorización feminista cobró mayor fuerza y se desarrolló sin pausa ni tregua alrededor del mundo, siendo la base que sustenta a movimientos y teorizaciones recientes como el feminismo islámico, negro, lésbico, chicano, indígena, gitano, transfeminista, interseccional, entre otros (2019).

Al margen de los debates externos e internos, que desde sus inicios han estado presentes en los estudios feministas, es posible contemplar dos premisas fundacionales: en primer lugar, estos elaboran una crítica al conocimiento convencional y a sus instituciones a fin de negar su autoridad, porque han ignorado o distorsionado la vida de las mujeres y sus contribuciones al conocimiento. En segunda instancia, estos estudios, toda vez que han sido analizados, tienen que pasar a una segunda etapa en la que se realice la reconstrucción del conocimiento, y de la cual sean las mujeres quienes se sitúen como sus edificadoras a partir de las diferentes disciplinas (Navarro y Stimpson, 1998).

En la década de los noventa, surge la polémica en torno al nombre del campo de estudio, lo que establece la dicotomía entre estudios feministas versus estudios de género, lo cual ha contribuido a la división entre las estudiosas que van desde el feminismo más radical hasta los estudios de género más academicistas. Veamos dos definiciones de los mismos. De acuerdo con Tong (2001), la teoría feminista es:

un conjunto de ideas que se originan en la creencia de que las mujeres no están subordinadas a los hombres o solo son valiosas en relación con los hombres (sirviente, cuidadora, madre o prostituta), y que las disciplinas, los sistemas y las estructuras existentes en nuestro mundo de hoy pueden cambiar para mejor si se infunde un punto de vista feminista. Además, la teoría feminista establece una agenda de acción, cuyo objetivo es la justicia y la igualdad para las mujeres en todas partes y, por supuesto, también para los hombres y niños con los que están indisolublemente vinculadas.

En esta definición destaca el hecho de que el feminismo pretende no solo estudiar, sino también cambiar la situación de las mujeres en el mundo. Por otra parte, la teoría de género se define como:

el estudio de lo que se entiende como comportamiento masculino y / o femenino y / o queer en cualquier contexto, comunidad, sociedad o campo de estudio dado...La diferencia entre sexo y género es que el primero se refiere a categorías del cuerpo humano biológicamente observable, femenino y masculino o intersexual, mientras que género se refiere a las categorías de expectativas sociales, roles y comportamientos, femenino y masculino (Jule, 2014).

Como puede verse, en la definición de estudios de género no prevalece como premisa fundante el cambio de la situación de las mujeres en el mundo, sino que es una situación que está implícita. Sin embargo, Tubert (2003) señala que el precio de ganar visibilidad académica nombrando al campo de estudios como "estudios de género", lo cual implica 
una mayor "neutralidad" y aceptación como campo científico, supuso un creciente divorcio entre la historia del género y la originaria historia feminista.

Para Mercedes Bengoechea (2003), por su parte, el divorcio entre la teoría feminista y sus aplicaciones, así como la popularización del concepto de género, tuvo dos consecuencias básicas: en primer lugar, destaca la asimilación de género a sexo, pues el simple cambio de términos afecta de manera considerable la manera de entender qué tipo de estudios se contemplan. En segunda instancia, se encuentra una creciente despolitización, ya que el uso de la categoría género supuso un mero cambio de etiqueta más que de postulados esenciales: las investigaciones seguían conceptualizando a la mujer y al hombre como categorías preexistentes, en lugar de categorías construidas en un contexto social de relación, como dictaba el paradigma.

En el análisis sobre la trayectoria de los estudios de género, se encontró que la producción inicial de la década de los 1960, y la segunda mitad de la década de los ochenta, representa un periodo de consolidación de la teoría de género. Desde entonces no sólo se han abierto centros académicos en todos los continentes, sino que la teorización se difunde y se legitima en campos tan variados que van desde la economía o la geografía, hasta la neurobiología, pasando por los profundos análisis de las disciplinas en las que desde un principio aparecieron los estudios feministas, como fueron la antropología, la psicología, la literatura y la historia. Así, pues, el eje analítico de la denuncia y la visibilidad anteriores ha adquirido una multiplicidad de diferenciaciones en intersección.

La complejidad de los usos, contenidos y supuestos que se han hecho del género en las disciplinas sociales y humanísticas, hacen sumamente difícil elaborar una clasificación y un análisis comprensivo del desarrollo teórico contemporáneo. Frente a este reto, en el presente artículo nos proponemos hacer un recuento de los principales desarrollos teóricos contemporáneos occidentales, en torno al feminismo y el género, para lo cual nos apoyaremos en la clasificación de la teoría feminista contemporánea propuesta por Madoo y Niebrugge-Brantley (2004), con las adaptaciones y adecuaciones que hemos considerado pertinentes. De esta forma, el esquema de análisis teórico es como sigue:

Figura 1. Principales teorizaciones en referencia al feminismo y el género
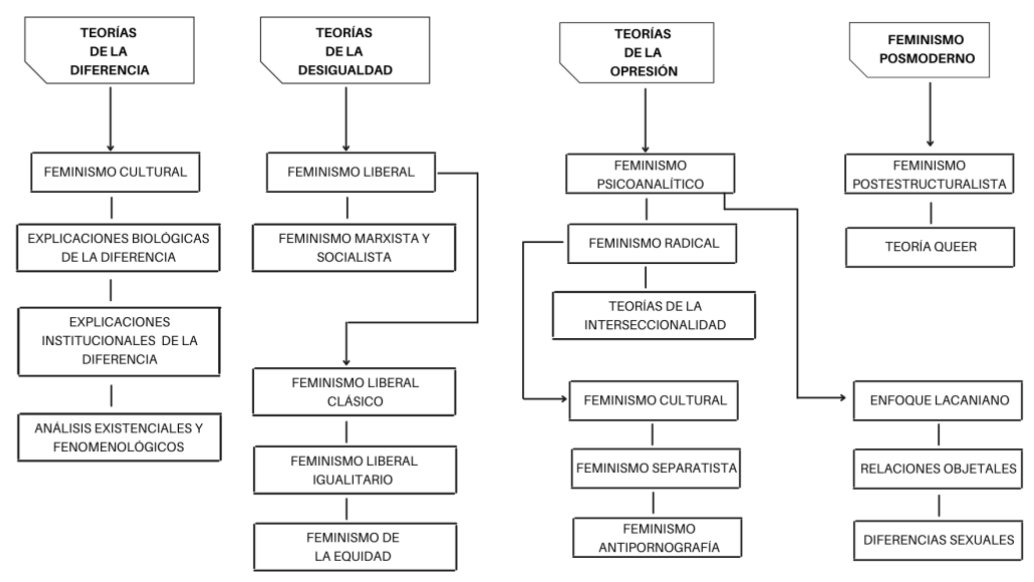

Elaboración propia con base en Madoo y Niebrugge-Brantley (2004). 
Las preguntas de investigación que guían este artículo son ¿es posible identificar etapas de desarrollo en el devenir histórico de las teorías feministas y de género a lo largo de los años? ¿Cuáles son las similitudes y diferencias entre las teorías estudiadas? ¿Hacia dónde va el feminismo?

Por otra parte, se parte del supuesto de que el patriarcado es un sistema histórico, es decir, tiene un inicio en la historia. Si es así, puede acabarse gracias al proceso histórico. Si el patriarcado fuera "natural", es decir, que estuviera basado en un determinismo biológico, entonces cambiarlo supondría modificar la naturaleza (Lerner, 1990). Esta afirmación alienta a todas las y los feministas a seguir trabajando en la teoría y en la práctica para lograr un mundo más equitativo y más justo para todos y todas.

\section{PERIODIZACIÓN HISTÓRICA DE LAS TEORÍAS FEMINISTAS Y DE GÉNERO.}

Toledo-Nickles (2008) sintetiza la función de las teorías para dar cuenta de la pluralidad fenoménica y su papel en la configuración de los campos del conocimiento. Los resultados de las investigaciones incrementan la información de la que se dispone y permiten contar con constructos teóricos cada vez más amplios y consistentes que robustecen las teorías hasta configurar paradigmas. Nos preguntamos entonces si esto ha sido el continuo de la teoría de género, que ha pasado por todas estas etapas y que requiere de su consolidación como paradigma mediante el robustecimiento de las teorías que lo conforman, o, por el contrario, cada vez habrá una mayor dispersión que lleva a tener distintos puntos de vista que puede o no, favorecer el desarrollo teórico. En tal sentido, el análisis de la teoría feminista y de género permite observar el siguiente continuo:

Figura 2. Proceso histórico de la teorización feminista y de género

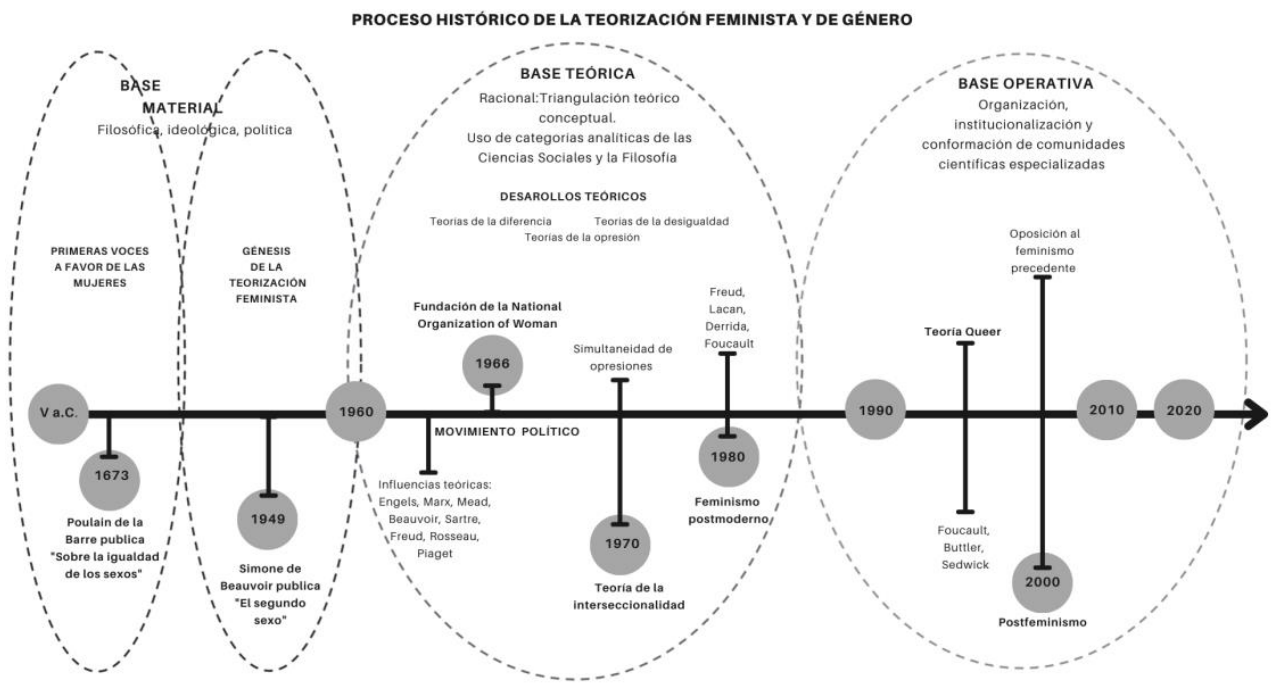

Fuente: Elaboración propia.

En este esquema es posible visualizar el devenir de las primeras voces a favor de las mujeres, que comienza con las reflexiones y el ejemplo vivo de inteligencia, creatividad y sapiencia de tres mujeres griegas Aspacia de Mileto, Safo e Hipatia de Alejandría, pasando a la Edad Media en donde se discurría si las mujeres tenían igual estatus espiritual e intelectual que los hombres, periodo de oscuridad para las mujeres por 
la persecución de mujeres acusadas de brujería excepto por la aparición de comunidades de beguinas, que compartían casa, trabajo, comida y vida sin ninguna autoridad masculina, y llegaron a tener miles de seguidoras en Alemania, Holanda, Francia, Italia, España, Polonia y Austria (Iglesias, 2003). En 1673, el libro de Poulain de la Barre "Sobre la igualdad de los sexos", constituye el primer escrito considerado feminista, escrito paradójicamente por un hombre, en el que el autor se centra explícitamente en fundamentar la igualdad sexual, abandonando la comparación entre los sexos masculino y femenino.

La génesis de la teorización feminista comienza con la publicación de "El segundo sexo" de Simone de Beauvoir, (2005) obra fundante para el feminismo, en la que la autora plantea que la mujer no nace, sino que llega a serlo por las condiciones emocionales, sociales y culturales prevalecientes con lo que se genera una serie de ideas y constructos teóricos que tienen su auge en 1960 cuando se inicia la formalización de la teorización feminista, en un marco de crisis política, social y cultural. Este periodo puede llamarse, siguiendo el esquema de Ibarra y Montaño (1990), Base Material, en el que se da una serie inicial de desarrollo de ideas y de acontecimientos políticos que propiciaron el surgimiento de teorías.

Llegó entonces el momento de pasar del feminismo como movimiento político y social a una reflexión más profunda en el plano teórico y a tomar posiciones en ese sentido, mediante la diversificación de las teorías. Este paso que podríamos llamar de transición del feminismo como movimiento político al feminismo como cuerpo teórico, constituye la Base Teórica y esta arranca con marcos explicativos que constituyen en realidad el principio del proyecto teórico de género. Apoyados en la Base Material, estos desarrollos teóricos inician con el trabajo empírico y la implementación metodológica que ello implica (Ver figura 2).

Aquí observamos que el pensamiento feminista echó mano de las herramientas teórico-conceptuales de las teorías humanistas y sociales más fuertes de las disciplinas sociológicas, antropológicas, psicoanalíticas y filosóficas, las cuales proporcionaron la base para el análisis desde una perspectiva de género. Partiendo de una plataforma multidisciplinar, la teorización permite el despegue de la investigación empírica que teniendo como base la perspectiva de género, busca visibilizar la situación diferenciada y desigual que viven hombres y mujeres. Esta etapa en la que se expandió la investigación empírica sería la Base Operativa.

La Base Operativa, se constituyó por el crecimiento exponencial y la amplia difusión de la investigación feminista y de género en universidades y centros de investigación, apoyada por la Base Teórica, que floreció en la década de 1960 y 1970, con una gran producción de autoras provenientes de campos tan divergentes como las ciencias humanas y sociales y las cognitivas, la genética, etc. que desde sus disciplinas hacen aportes y enriquecen la teorización y la base empírica. En esta etapa, con una base material y teórica, es posible el desarrollo de nuevas teorías feministas y de género que multiplican las miradas de la situación de las mujeres en el mundo, así como de las relaciones intergenéricas y los problemas que estos acarrean en los planos institucionales, culturales y personales.

Con el surgimiento de nuevas teorías que tienen como origen los desarrollos teóricos que se van a revisar en este artículo, prevalece un cierto aislamiento entre corrientes disímiles lo que dificulta un programa de investigación común en género. Como plantea Acker: "no existe un entendimiento común de su significado, incluso entre las académicas feministas" (Acker 1992:565). Mientras tanto, la producción a nivel 
empírico sigue siendo prolífica y se han anexado los estudios sobre masculinidades, así como los feminismos específicos a ciertas culturas como sería el feminismo islámico, el feminismo chicano o indígena, el decolonial, entre otros.

Aunque en la práctica los términos teoría feminista y teoría de género se usan de forma indistinta, y pertenecen a una misma tradición científico-académica, hemos podido identificar a través de la revisión de las principales tradiciones teóricas, que aun cuando existen claras diferencias entre las teorías feministas revisadas, éstas comparten sus supuestos centrales. Sin embargo, entre las teorías feministas y aquellas que pretenden ser teorías de género, hay sutiles distinciones a nivel ontológico, epistémico y metodológico. En este sentido, Benhabib y Cornell reconocen que el antecedente directo de la teoría de género es el feminismo y sus teorías:

Como paradigma ético inédito, como visión filosófica contemporánea, como óptica renovada para comprender el desarrollo de la historia, y como acción política democratizadora, la Teoría de Género tiene su punto de partida en el

\section{METODOLOGÍA}

feminismo contemporáneo (Benhabib y Cornell, 1990: 9-28).

El enfoque metodológico utilizado en esta investigación teórica es el de la Metateorización propuesta por George Ritzer (1992), la cual tiene la finalidad hacer una revisión de dos o más teorías con el fin de hacer una crítica meticulosa y una revisión profunda a todos los niveles para desarrollar y expandir la teoría. Ritzer advierte que la metateorización no es un asunto sencillo de realizar, por un lado, por el nivel de profundización y conocimiento de la teoría que esto implica, pero también por el hecho de que al hacer una metateorización en un campo de estudios particular, se pone sin querer, el dedo en la llaga, al hacer una crítica profunda y amplia tanto de la producción en sí misma como de las circunstancias sociales que la rodean.

Ritzer distingue tres orientaciones principales de metateorización en sociología (Ritzer 2002:602-606): 
Figura 3. Orientaciones principales de la metateorización según George Ritzer.
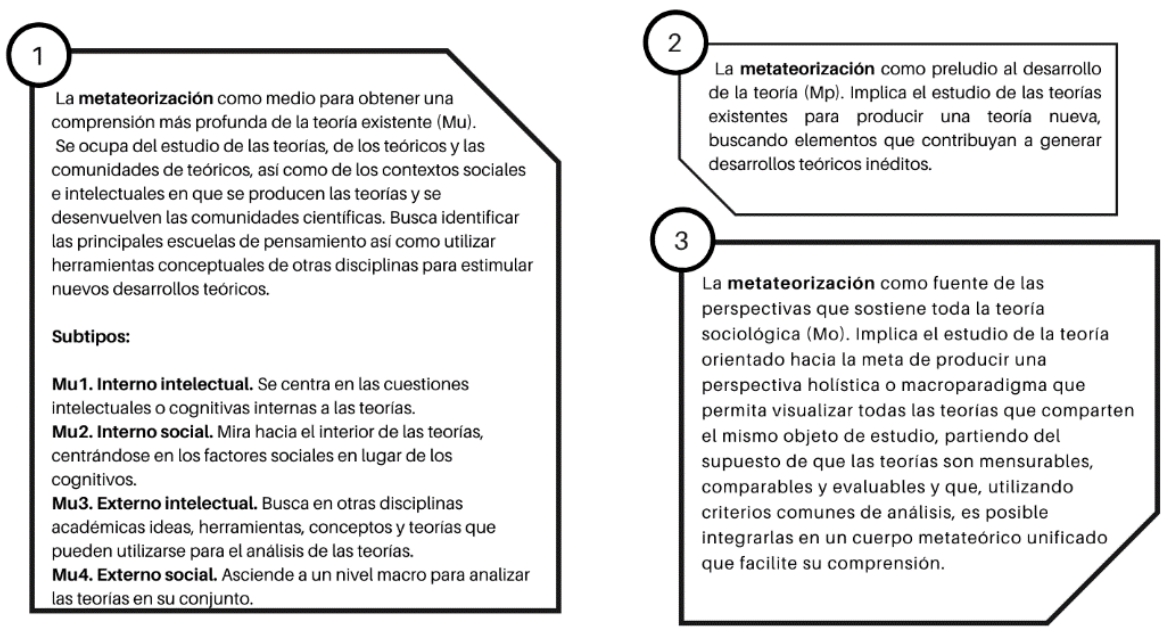

Fuente: Elaboración propia a partir de Ritzer (2002:602-606)

Aunque la propuesta de Metateorización de Ritzer se aplica a las teorías sociológicas, también es posible utilizarla desde un punto de vista metodológico para analizar las teorías feministas y de género y hacer una metateorización. En el caso de este artículo, se ha realizado una metateorización del tipo Mu, mediante la revisión de las teorías, las teóricas, sus comunidades de pertenencia, así como el contexto socio cultural del que surgieron.

\section{TEORÍAS FEMINISTAS/TEORÍAS DE GÉNERO}

A continuación, se hace una breve síntesis de las principales teorías feministas y teorías de género que se han desarrollado en Occidente, las cuales dejan ver la complejidad del tema, en donde se construyen y se deconstruyen las binariedades femenino/masculino; hombre/mujer; naturaleza/cultura; dualismo/pluralismo entre otras. Debido a las limitaciones de espacio, se analizarán las características generales de las corrientes: Teorías de la desigualdad, Teorías de la diferencia, Teorías de la opresión y Teorías Psicoanalíticas, para pasar a revisar con mayor exhaustividad las siguientes teorías: feminismo liberal, feminismo radical, las teorías de la interseccionalidad, el feminismo posmoderno y la teoría queer, debido al impacto que han tenido en los desarrollos teóricos en el siglo XXI. 


\subsection{TEORÍAS DE LA DIFERENCIA}

Existen dos argumentos dentro de los estudios feministas: uno enfatiza cómo las mujeres construyen, a partir de su propia experiencia individual, diferentes formas de entender el mundo en comparación con las de los hombres; el otro da cuenta del "ataque" que han realizado aquellos hombres que insisten en considerarlas inferiores por ser diferentes. En este sentido, para las teorías de la diferencia, la localización de las mujeres y su experiencia en diferentes situaciones difiere de la de los hombres y por ello buscan preservar una contracultura que exalta lo femenino y denigra lo masculino (De Miguel, 2007)

\subsection{TEORÍAS DE LA DESIGUALDAD}

En contraste con las teorías de la diferencia, las teorías de la desigualdad de género consideran que, en la mayoría de las situaciones sociales, las mujeres tienen una posición no sólo diferente, sino desigual y menos privilegiada, en relación con la de los hombres. La estadística da cuenta de que mujeres con idéntica posición social, nivel educativo, etc. tienen menos recursos materiales y estatus social que los hombres.

\subsubsection{FEMINISMO LIBERAL}

El feminismo liberal afirma que es posible lograr la igualdad de hombres y mujeres mediante una reforma política y jurídica; así pues, vemos que su antecedente histórico más importante es la Declaración de Sentimientos, publicada en 1848, la cual resulta de la Primera Convención de los Derechos de la Mujer realizada en Seneca Falls, Nueva York (Declaración de sentimientos, 2021). En este documento se reclamaban para las mujeres los mismos derechos concedidos a todos los seres humanos, de acuerdo con la ley natural. Un antecedente aún más tardío son los escritos de Mary Wollstonecraft (2014), quien publicó en 1792 La vindicación de los derechos de la mujer, escrito en el que aboga por los derechos de las mujeres y hace patente la opresión sexual que sufren.

Para esta corriente, la desigualdad de género es el resultado de un modelo sexista y patriarcal de la división del trabajo, donde se excluye a las mujeres de la esfera pública; por tal motivo, una de sus principales demandas era la inclusión femenina en el mercado laboral así como en puestos públicos, señalando asimismo que el trabajo doméstico no debe recaer únicamente en ellas, pues es causa de estrés.

Bety Friedan, quien es considerada como la líder histórica del feminismo liberal, fundó en Estados Unidos, la Organización Nacional para las Mujeres (NOW, por sus siglas en inglés), en el año de 1966. Esta institución, considerada como emblemática del feminismo liberal, actualmente continúa teniendo influencia política y académica; sobre todo trabaja en favor de la erradicación de prejuicios institucionales y vigila la aplicación de mejores leyes.

El feminismo liberal alberga las siguientes ideas (Fundación para el progreso, 2021): considera que mujeres y hombres son igualmente dignos, independiente de su sexo, condición o convicciones; cree en el entendimiento y la complementariedad de hombres y mujeres y es contrario a la idea de "lucha de géneros"; fomenta una cultura de autonomía y responsabilidad por sobre una cultura de la victimización; rechaza la violencia en todas sus formas; anhela la justicia sin privilegios y la igualdad de todos los individuos ante la ley; cree que la identidad y derechos son individuales, no colectivos; promueve que cada mujer sea libre para seguir sus proyectos de vida; sostiene que el libre mercado es el mejor aliado para la emancipación, progreso y desarrollo de la mujer; reconoce la evidencia científica respecto a las diferencias entre hombres y mujeres; defiende la tolerancia, el respeto y la diversidad entre individuos y sociedades mediante el diálogo que se contrapone a las actitudes intolerantes. 
Para concluir, es necesario mencionar que los argumentos del feminismo liberal han sido duramente cuestionados por feministas de otras adscripciones filosóficas y políticas, debido a que los relacionan con el capitalismo propio de los países desarrollados; además, no es posible generalizarlos en un plano global, ya que son muchos más los países en los que las mujeres viven en condiciones de desigualdad. Las soluciones que proponen, de acuerdo con sus críticas, no son ni simples ni viables, pues hay otros factores históricos, ideológicos, culturales, etc., que se encuentran profundamente arraigados en hombres y en mujeres que las impiden.

\subsection{TEORÍAS DE LA OPRESIÓN}

Las teorías de la opresión de género afirman que las mujeres son diferentes a los hombres, pero que, además, se encuentran en peores condiciones sociales, económicas, etc. en comparación con ellos. Para estas teóricas, las mujeres están oprimidas, subordinadas, reprimidas, moldeadas e incluso son objeto de abuso y maltrato por parte de los hombres.

\subsection{FEMINISMO RADICAL}

Entre los años 1967 y 1975 se desarrolló el feminismo radical, el cual le da un tinte mucho más político a la teoría feminista desde su lema "lo personal es político" y mediante el concepto de la "política de la experiencia". Estas ideas implicaban que, desde las experiencias vividas se tenía que teorizar y hacer política. Las manifestaciones políticas de las feministas radicales fueron muy espectaculares en su época; tuvieron mucho eco en la teorización y en la práctica feminista, así como en la sociedad en general.

Las dos obras más influyentes del feminismo radical fueron Política Sexual de Kate Millet (1995), publicada en 1969; y La dialéctica del sexo de Shulamit Firestone (1976), publicada por primera vez en 1970. En estas obras se definieron conceptos fundamentales para el análisis feminista: patriarcado, género y casta sexual, los cuales constituyen el sistema básico de dominación sobre el que se levantan el resto de las dominaciones como la clase y la raza. El patriarcado, siguiendo esta corriente, es un sistema de dominación masculina que determina la opresión y subordinación de las mujeres; el género expresa la construcción social de la feminidad y la casta sexual se refiere a la experiencia común de opresión vivida por todas las mujeres (Varela, 2005).

La diferencia sustancial entre el feminismo radical y otras teorías feministas radica en el interés por la sexualidad y la familia dentro del patriarcado que, para las radicales, es el centro de la dominación masculina. Para acabar con él, consideran necesario explorar la parte íntima y personal, así como transformar las relaciones de poder, lo cual implica ir más allá de las propuestas de las feministas liberales que abogan por ganar espacios públicos, alcanzar una igualdad en el trabajo, en la educación o en los derechos civiles y políticos.

El feminismo radical se basa en dos creencias centrales: primero, las mujeres tienen un valor positivo absoluto; segundo, se encuentran violentamente oprimidas por el sistema patriarcal. Bajo dicho sistema, todos los hombres, independientemente de su clase social, identidad étnica o raza, reciben beneficios económicos, sexuales y psicológicos de las mujeres; para llegar a la raíz de la dominación masculina, es necesario comprender que la fuente de poder es la sexualidad masculina y que en el coito heterosexual se promulga la dominación (McAffee, 2016).

Las feministas radicales piensan que en el patriarcado, el poder se da de forma vertical, en un solo sentido: parte de los que tienen poder y se dirige a los que son 
oprimidos, de ahí que McKinnon (1989: 113, citada por McAffee, 2016) señale lo siguiente:

Las mujeres y los hombres se dividen por género, de acuerdo con la división de los sexos que conocemos, lo cual deviene de los requerimientos de la heterosexualidad como forma dominante, la cual institucionaliza la dominación sexual masculina y la sumisión femenina. Si esto es cierto, la sexualidad es el eje de la inequidad de género.

Las radicales creen y consideran que los hombres mantienen el patriarcado no sólo porque tienen los recursos para tal fin, sino porque las mujeres, sin saberlo, constituyen la fuente de satisfacción de múltiples necesidades: en el ámbito sexual constituyen signos ornamentales que reflejan el estatus y el poder del hombre; con la procreación, ellas se convierten en protectoras, compañeras y fuentes de apoyo emocional tanto para los hijos como para los varones adultos. En este estado de cosas, las mujeres pasan a ser una especie de sujetos de contraste que refuerzan continuamente la percepción de los varones de que son ellos quienes tienen un significado social central.

Las feministas radicales consideran que el patriarcado explica la violencia contra las mujeres; y ésta no sólo se manifiesta a través de la violencia física, sino también de manera oculta mediante prácticas más complejas de explotación y control; por ejemplo, en criterios de moda y belleza, en la idealización de la maternidad, la monogamia, la castidad, la heterosexualidad, el acoso sexual, el fatigoso trabajo doméstico no pagado y los menores salarios pagados a las mujeres en el trabajo asalariado (Madoo y NiebruggeBranthey, 2002).

Existen diferencias entre las feministas radicales respecto a la respuesta más apropiada para acabar con el patriarcado. Algunos grupos sostienen la necesidad de instaurar un matriarcado compensatorio; otros prefieren la formación de fraternidades igualitarias femeninas. El feminismo radical se caracteriza por considerar la lucha política contra la opresión como una condición indiscutible para lograr cambios que lleven a la emancipación de las mujeres (Barry, 2005). En lo académico desarrollos posteriores del feminismo radical llevaron a reconocer la intersección de otras categorías de opresión: la identidad de género, la raza, la clase social, la sexualidad, la orientación sexual, y la capacidad intelectual.

\subsection{TEORÍAS DE LA INTERSECCIONALIDAD}

Las teorías de la interseccionalidad parten del imperativo teórico y estratégico de considerar que las mujeres experimentan la opresión en varias formas y en distintos grados de intensidad, y que existe una intersección de variables de opresión que es distinta en cada individuo (Cobo, 2000).

Esta teoría considera que la sociedad tiene dispositivos de desigualdad social, los cuales constituyen vectores de opresión y privilegio; entre ellos destacan no sólo el género, sino también la clase, la raza, la situación geográfica, la preferencia sexual y la edad. La variación de estas intersecciones altera cualitativamente la experiencia de ser una mujer, lo que debe tomarse en cuenta para teorizar y dar cuenta de sus experiencias. Desde esta perspectiva, cruzar variables de opresión es un imperativo sociológico y político. Sin embargo, el dilema al que lleva la teoría interseccional es a que si es posible considerar al patriarcado como una estructura transcultural de dominio o bien la discriminación es local y contextual (Cobo, 2000).

Rita Mae Brown (1974) señala que la identidad de una mujer de clase obrera se basa no sólo en su relación con la producción, sino en las experiencias básicas de su infancia. Molyneux (1984), por su parte, dice que no existen "los intereses de la mujer" 
en abstracto que puedan unificar a las mujeres en la lucha política; más bien, tienen intereses prácticos de género que desarrollan a causa de la división sexual del trabajo.

La intersección de vectores de opresión y privilegio crea variaciones en las formas y en la intensidad con la que las personas experimentan la opresión. Las intersecciones que más estudian las feministas son las de género y raza; género y clase; raza, género y clase, aunque hay otros análisis que estudian el género y la edad; el género y la situación geográfica; así como el género y la preferencia sexual.

Entre las preocupaciones centrales de esta teoría está el cómo defender el principio analítico y el hecho empírico de la diversidad entre las mujeres, al mismo tiempo, la posición política y valorativa de que determinados grupos de mujeres comparten un punto de vista distintivo. Patricia Hill Collins (1998) propone la categoría de punto de vista como una visión compartida por un grupo que tiene una comunalidad heterogénea; en términos marxistas, esto quiere decir que aun cuando tienen vectores de diversidad, las mujeres comparten circunstancias directamente vividas, dadas y transmitidas por el pasado.

\subsection{FEMINISMO POSMODERNO}

Durante la década de 1990 algunas feministas empezaron a incorporar las ideas y el vocabulario posmoderno en su trabajo teórico; sin embargo, se han mostrado cautelosas en lo que respecta a la teoría social posmoderna; por un lado, consideran que está demasiado alejada de las luchas políticas y que puede llevar a las personas a permanecer fuera de la acción colectiva o conducirlas hacia un individualismo radical; por otro, porque sostienen que el posmodernismo está demasiado divorciado de la realidad material, se centra únicamente en el discurso, la representación y los textos, ignorando los hechos fácticos como la desigualdad, la injusticia y la opresión, lo cual lleva a pensar a autoras como Benhabib (2005), a que existen diferencias irreconciliable que favorecen una ruptura.

El feminismo posmoderno intenta construir conversaciones entre tres vertientes importantes del discurso occidental contemporáneo: el psicoanálisis, la teoría feminista y la filosofía posmoderna, lo que le permite buscar articulaciones entre los problemas concernientes al conocimiento, la diferencia entre los sexos, la subjetividad y el poder (Tubert, 2009).

Dentro del feminismo posmoderno ubicamos al posestructuralismo feminista de la escuela francesa, donde se encuentran Luce Irigaray (2007); Julia Kristeva (1997) y Hélène Cixous (1991), autoras que se estudiarán desde la vertiente filosófica de sus discursos. Otros nombres relevantes son: Linda Alcoff (2002); Toril Moi (2001); Sandra Harding (1996); Jane Flax (1995); Teresa de Lauretis (1992,1991); Donna Haraway (1991, 1989); Nancy Fraser (1990), entre otras, quienes cuestionan el discurso de la razón en cuanto a su posición universalista; su crítica está dirigida a la cultura occidental en cuanto a sus límites estrechos y poco accesibles; por lo tanto, encuentran que el paradigma posmoderno es mucho más conveniente para realizar las aproximaciones feministas sin tener que atenerse a los cánones establecidos (Hernández, 2003).

Uno de los elementos comunes en las corrientes posmodernas feministas es el posracionalismo, el cual plantea que el feminismo debe iniciar una ruptura profunda con el paradigma racionalista para ofrecer nuevas narrativas que sean descentradas y parciales, en donde los supuestos del humanismo de género y el feminismo construido como una teoría y una política para el sujeto mujer ya no existan. 
La filosofía posmoderna pone en evidencia la conciencia de que el progreso científico y tecnológico, el derrumbe de sistemas ideológicos y los estragos producidos por las economías de libre mercado, tienen unos elevados costos sociales que han opacado los referentes éticos y políticos. Benhabib (2005), basándose en varias autoras que han analizado la compleja relación entre feminismo y posmodernidad, propone una analogía entre las tres tesis centrales de la posición posmoderna y su versión feminista, en la que se destacan los siguientes puntos de unidad y diferencia:

La muerte del hombre vs. desmitificación del sujeto masculino de la razón. Una de las características de los posmodernos es el afán de destruir todas las concepciones existencialistas del ser o de la naturaleza humana. En esta perspectiva, el hombre se considera como un artefacto social, histórico o lingüístico, no un ser noumenal o trascendental.

Las feministas posmodernas están de acuerdo con esta postura que niega la existencia de seres absolutos, neutros, trascendentales e incognoscibles, pero agregan que la filosofía occidental, desde Platón y Descartes hasta Kant y Hegel, ha postulado al sujeto masculino, considerado neutro y universal, como el sujeto de la razón, deslegitimando y ocultando la presencia de lo otro y de la diferencia, que no encaja en sus categorías, es decir, del sexo femenino.

La muerte de la historia vs generización de la narración histórica. Para los posmodernos, la idea de que la historia tiene su propio ser o existe por sí misma es algo más que otro mito del concepto del progreso. El progreso, en sí mismo, es una parte muy importante de la historia del hombre y presupone los valores de unidad, homogeneidad, totalidad, clausura e identidad. De allí que los posmodernos hablen de la muerte de la historia.

Para la versión feminista posmoderna, si el sujeto de la tradición intelectual occidental ha sido normalmente el cabeza de familia varón, blanco, propietario, cristiano, entonces la Historia tal como se recuerda y se narra hasta ahora ha sido "su historia", pues se niega la fragmentación, heterogeneidad y sobre todo, las temporalidades variables que experimentan los distintos grupos no hegemónicos. Hasta hace muy poco las mujeres han recuperado su propia historia mediante el trabajo histórico hecho desde la perspectiva de género.

La muerte de la metafísica vs escepticismo feminista hacia las pretensiones de la razón trascendental. Para los filósofos posmodernos la metafísica ha muerto; para ellos no existe nada que esté más allá de lo real, lo histórico y lo particular. Frente a las contradicciones y desacuerdos, el feminismo y las feministas se encuentran ante una encrucijada: si abandonan el proyecto ilustrado al que en realidad nunca pertenecieron y se identifican con el proyecto posmoderno de la deconstrucción, podrían desaparecer conceptos tales como ciudadanía, derechos humanos, igualdad y justicia que, por muy limitados y ambiguos que sean sus beneficios, no han sido disfrutados por la totalidad de la humanidad, y sobre todo, por las mujeres.

Una de las teóricas que ven al pensamiento posmoderno como un peligro para el feminismo es Di Stefano (1990), quien señala que el posmodernismo expresa los intereses y pensamiento de un sector específico de la sociedad: el de los hombres blancos, privilegiados, del occidente industrializado que ya tuvo su ilustración pero que ahora quiere someterla a un escrutinio crítico, sin tomar en cuenta a los millones de mujeres que viven en países y zonas geográficas pobres que no han resuelto sus necesidades básicas y se encuentran en un nivel de sobrevivencia, lo que no les permite darse el lujo de pensar en deconstruir una ilustración que ni siquiera han tenido. 


\subsection{TEORÍA QUEER}

La teoría queer tiene como objeto de estudio la identidad sexual de los individuos; a nivel político tiene como precedentes los movimientos de homosexuales y lesbianas, conocidos como LGBTQ (Lésbico, gay, bisexual y transexual y queer, por sus siglas en inglés). Su antecedente teórico más importante son las teorías feministas; sin embargo, es posible encontrar referencias de esta teoría en estudios de corte médico, biológico, antropológico, filosófico y literario.

Desde la biología, los trabajos del médico y sexólogo alemán Magnus Hirschfeld (2000), considerado como uno de los fundadores del estudio científico del sexo, constituyen uno de los antecedentes más importantes de la teoría queer. Este científico y activista buscó examinar desde un punto de vista objetivo, la homosexualidad, el travestismo y la identidad de género, llegando a distinguir la homosexualidad auténtica de la falsa, así como el carácter innato de la homosexualidad y bisexualidad. De igual forma, rebate la teoría de que la homosexualidad es tratable.

Por otra parte, uno de los primeros materiales empíricos que sirvieron de sustento para la teoría queer es el ensayo Sexo y temperamento en tres sociedades primitivas, de Margaret Mead (2006). La autora llega a la conclusión de que los distintos rasgos psicológicos atribuidos al temperamento femenino y al masculino no constituyen el producto de un determinismo biológico, sino que derivan de una particular estructura cultural y que por tanto, hay sociedades en donde los roles de género se intercambian, de ahí que no puede decirse que son de alcance universal.

Otra influencia primordial para esta teoría son los estudios filosóficos y literarios posmodernos que descentran al sujeto, bajo el argumento de que las facultades intelectuales y espirituales, así como los roles sociales que asumen los varones y las mujeres, son el resultado de una multiplicidad de procesos de socialización, mas no de su herencia biológica. Un ejemplo de ello se encentra en la Historia de la Sexualidad, de Foucault (2005), en la que señala la libertad concedida al deseo en un estado de naturaleza y la represión sexual ejercida en las civilizaciones avanzadas.

Los estudios literarios constituyen otro antecedente preponderante en la teoría queer, dado que analizan la manera en que los textos dan atributos de normalidad a la heterosexualidad, mientras que a todas las demás formas de sexualidad se les considera como perjudiciales, inadecuadas o anómalas.

La teoría queer afirma que la orientación sexual y la identidad sexual o de género son volátiles y lábiles, son el resultado de una construcción social; por tanto, no existen roles sexuales esenciales o biológicamente inscritos en la naturaleza humana, sino formas socialmente variables de desempeñar uno o varios papeles sexuales. La identidad, de acuerdo con la teoría queer, se considera provisional, lo cual quiere decir que las identidades tienen una naturaleza efímera. La identidad queer no necesariamente se atribuye a mujeres o hombres que se definen como gays o lesbianas, sino a cualquier persona que en algún momento de su vida se han sentido fuera de lugar ante las restricciones de la heterosexualidad y de los papeles de género.

Uno de los textos claves de la teoría queer es Epistemología del armario, de Eve Kosfsky Sedwick (1990). La autora se manifiesta en favor de la centralidad absoluta de la sexualidad para la comprensión de la cultura moderna, pues para que entendamos cualquiera de sus aspectos, es necesario hacer un análisis crítico de la definición homo/heterosexual. La teoría queer establece una separación entre género y sexo, dado que por ejemplo, en el caso de los transexuales, el cambio de sexo implica la adquisición de una identidad que no se define exactamente como masculina o femenina, más bien 
como neutra. En este sentido, Butler (2002) señala que el sujeto se hace, se construye social, cultural y lingüísticamente como individuo generizado, diferente; pero dado que el sexo es ya género y que no existe ninguna identidad previa al trabajo de lo cultural, se le da el carácter performativo del género.

Mayobre (2006) señala que para Butler, tal performatividad comienza desde el momento que nacemos (incluso antes), desde que se nos asigna el sexo, el cual debe copiar los comportamientos, deseos y sensaciones propios de tal sexo, lo que hace que el sexo sea algo normativo desde sus inicios. La feminidad o masculinidad no es, en consecuencia, fruto de una elección, sino la cita o repetición forzosa de una norma cuya compleja historicidad es inseparable de las relaciones de disciplina, regulación y castigo. No hay "nadie" que escoja una norma de género, al contrario, la cita de las normas genéricas es necesaria para que tengamos derecho a ser "alguien".

La teoría queer se caracteriza por una contradicción que ha estado presente desde sus inicios en las teorías y prácticas feministas: la lucha entre la academia y la militancia. En el caso de la teoría queer es muy clara la diferencia entre ambas pues como teoría, más que de la militancia y el feminismo, se nutre de fuentes científicas, de teorías que pretenden explicar y fundamentar las diferencias entre los géneros, la neutralidad y la elección, apoyándose en distintas ciencias como la biología, la medicina y la psicología.

En este sentido Wilchins (2004) abre un debate en su libro Queer Theory, Gender Theory (Teoría Queer, Teoría de Género), ya que señala a los teóricos universitarios de haber "robado" la teoría queer a las clases populares; los acusa de utilizar un lenguaje muy elaborado, casi incomprensible, con el fin de compensar el estatus de inferioridad que se les adjudica dentro del mundo académico.

\subsection{HACIA UNA METATEORIZACIÓN DE LAS TEORÍAS FEMINISTAS Y DE GÉNERO.}

Después de haber hecho un análisis histórico y una revisión de las principales teorías feministas y de género, es posible plantear los puntos en común y las diferencias que hay entre una y otra. Como primer punto, encontramos que respecto a la visión de lo que es el feminismo y sus objetivos, el feminismo liberal considera que la igualdad entre mujeres y hombres puede lograrse mediante una reforma política que brinde iguales derechos o inclusive más derechos a las mujeres, a fin de equilibrar su situación en el mundo. En cambio, el feminismo radical parte de una postura en la que se exacerban las experiencias individuales del cuerpo y se aboga por considerar lo personal como político, teniendo un fuerte componente de lucha y activismo. En un sentido más complejo y abarcador, el feminismo de la interseccionalidad plantea que las mujeres experimentan la opresión en varias formas y grados de intensidad, los cuales hacen que una mujer sufra un mayor o menor nivel de opresión, dependiendo de variables como la clase, la etnia o la edad.

Por su parte el feminismo posmoderno se aparta de las anteriores versiones feministas e intenta construir conversaciones entre el psicoanálisis, la teoría feminista y la filosofía posmoderna para llegar a concluir que no es posible definir ni a la mujer ni al hombre pues las identidades son resultado del discurso que deviene en un mecanismo de poder que constriñe a los sujetos a una cierta identidad, por lo que definir a la persona en femenino no es una solución para erradicar el sexismo. Finalmente, la teoría queer se interesa por la identidad de los individuos, que, de acuerdo con esta teoría, se considera provisional, lo cual quiere decir que las identidades tienen una naturaleza efímera, abarcando también como queer, a cualquier persona que en algún momento de su vida se 
ha sentido fuera de lugar ante las restricciones de la heterosexualidad y de los papeles de género.

En suma, el objetivo del feminismo en todos los casos analizados (feminismo liberal, feminismo radical y feminismo interseccional), excepto por el feminismo posmoderno y la teoría queer, establece que las mujeres se encuentran en una posición desigual y subordinada a los hombres, por lo que buscan, por distintas vías, lograr la equidad de derechos y privilegios entre los géneros. Por su parte el feminismo posmoderno deconstruye la subjetividad en femenino y masculino, planteando que no puede haber definiciones claras y definitivas para ambos, en tanto que la teoría queer va más allá de la discusión entre los privilegios y la inequidad de dos géneros, estableciendo que hay más de dos maneras de vivir en el mundo y que la heteronormatividad patriarcal es la responsable de que solo se considere como "normal" la identidad femenina y masculina.

Respecto al sexo, el feminismo liberal considera que las mujeres tienen una posición desigual y menos privilegiada que los hombres, por ello es necesario emprender políticas públicas, tal como las acciones afirmativas, para lograr subvertir esta situación. En cambio, el feminismo radical otorga un valor positivo absoluto a las mujeres, en oposición a los hombres, que, en un punto de esta teorización, fueron considerados como excedentes e indeseables en la sociedad, buscando la posibilidad de la partenogénesis como solución para disminuir la población masculina en el mundo y para hacerlos innecesarios. La teoría interseccional por su parte, destaca que no existen los intereses de la mujer, sino más bien intereses prácticos de género, es decir, no existe "la mujer" en general, sino mujeres de carne y hueso, con sus especificidades dadas por las diferentes condiciones de opresión que viven, las cuales demandan particulares formas de lucha. En cambio, para el feminismo posmoderno, el sujeto masculino es neutro y universal, considerado el sujeto de la razón, deslegitimando "lo otro" que es la mujer. Respecto al sexo, la teoría queer asume el carácter innato de la homosexualidad y la bisexualidad, las cuales no son tratables por lo que denuncian la heteronormatividad como propia de la historia humana que se vive hasta el momento actual.

En tal sentido, vemos que nuevamente las cinco corrientes analizadas difieren en su concepción de sexo, siendo los planteamientos posmodernos y los de la teoría queer quienes se alejan más del objetivo del feminismo de lograr alcanzar la igualdad entre hombres y mujeres, centrándose más en los individuos que en una lucha social a favor de la equidad. La teoría queer visualiza a los individuos como capaces de tener distintas identidades a lo largo de su vida. Sin embargo, esta postura plantea retos para la lucha por la igualdad y la equidad, pues ¿de qué género se está hablando? ¿Cuál es el género oprimido?

En cuanto al género, las distintas corrientes feministas coinciden en el objetivo de la equidad de género, pero no en las formas: para el feminismo liberal la inclusión femenina en el mercado laboral y la igualdad de oportunidades educativas y sociales, logrará que se dé un equilibrio en la disparidad de género. En cambio, el feminismo radical ve a la sexualidad y la familia como el centro de la dominación masculina y es necesario hacer cambios en ambas para lograr que las mujeres salgan de la opresión en la que están envueltas. Para el feminismo interseccional, existen diversos vectores de opresión y privilegio, género, clase, raza, situación geográfica, preferencia sexual y edad y todos en conjunto conforman a las mujeres y los hombres de forma particular, por lo que no es posible hacer definiciones que generalicen qué es una mujer o qué es un hombre ni tampoco las necesidades de género particulares. En el feminismo posmoderno, 
no existen seres absolutos, neutros, trascendentales e incognoscibles, sino que estos están en constante construcción y deconstrucción, lo que dificulta una lucha por eliminar los sesgos del género ya que no constituyen el objetivo central del feminismo posmoderno. Por su parte, la teoría queer concibe la orientación sexual y la identidad sexual o de género como volátiles y lábiles, son el resultado de una construcción social y su lucha se circunscribe a la defensa de los derechos del abanico de identidades queer.

Respecto al patriarcado, el feminismo liberal considera que se va a erradicar cuando ambos sexos tengan igualdad de derechos y de condiciones para romper los techos de cristal que prevalecen sobre las mujeres, por lo tanto, es un tema de política y de instituciones. Para el feminismo radical el patriarcado es un sistema básico de dominación universal que se va a trascender a través del proceso de naturalización de las mujeres oprimidas, un análisis de la sexualidad que desembocará en una crítica a la heterosexualidad obligatoria, la denuncia de la violencia patriarcal, que no solo es la sexual y finalmente una sociología del conocimiento que será crítica al androcentrismo en todos los ámbitos, incluidos los de la ciencia (Puleo, 2021).

Según el feminismo interseccional, no existen estructuras globales de dominio tales como "patriarcado" o "capitalismo", sino formas sociales locales y contextuales de discriminación que son una resultante del conjunto de vectores de opresión que sufren las mujeres en particular. En el caso del feminismo posmoderno, los hombres sostienen el patriarcado no sólo porque tienen los recursos para tal fin, sino porque las mujeres son fuente de diversos satisfactores para sus necesidades: sexuales, reproductivas y en el del mantenimiento emocional de niños y hombres. Para la teoría queer, la heteronormatividad es producto del patriarcado y precisa que la sociedad la deconstruya para vivir en un mundo en que todas las identidades sexuales puedan ser libres y aceptadas.

\section{CONCLUSIÓN}

Hacer una metateorización de las teorías feministas y de género no es una tarea fácil, menos aún cuando se pretende abarcar un largo periodo de tiempo y una variedad de teorías que han emergido y se han sostenido con el paso de los años, transformándose y adecuándose a las nuevas realidades y necesidades. Los estudios feministas y de género se han institucionalizado en el periodo que se denominó base operativa (ver figura 2), forman parte de los cursos académicos de grado y posgrado en la mayor parte de universidades, centros de investigación y países del mundo occidental. La teoría feminista es rica tanto en sus temas de estudio como en las perspectivas ontológicas, epistemológicas y metodológicas de las que parten para acercarse a sus temas de interés.

Los planteamientos de la teoría queer que revelan y legitiman la existencia de más de dos géneros viene a complejizar los análisis precedentes de las teorías feministas que se ocupaban de la relación binaria hombre-mujer. Hoy por hoy lo queer atraviesa medios de comunicación de masas, redes sociales, industria cinematográfica, etc., mientras que en sus comienzos esta teoría se estigmatizaba como lo otro que no encajaba del todo en la teorización existente. Por otro lado, los aportes de la teoría de la interseccionalidad dan cuenta de que las opresiones que sufren las mujeres no pueden generalizarse, pues las distintas variables de opresión que atraviesan sus vidas las hacen diferentes a unas de otras y lo que las mujeres tienen en común es el objetivo de encontrar vidas vivibles (Butler, 2004), dentro de los parámetros de las variables que las condicionan, pero que en definitiva pueden romperse como techos de cristal. 
Cabe señalar también que, en la segunda década del siglo XXI, el feminismo es para muchas y muchos jóvenes, un tema ya superado, estamos hablando del postfeminismo que se expresa de múltiples formas en las redes sociales, los medios de comunicación y la vida cotidiana (Banet-Weiser, 2018). Muchas jóvenes se preguntan por qué seguir hablando de feminismo cuando ya se han obtenido derechos, sin darse cuenta de que están sentadas sobre los hombros de las feministas del pasado y aún del presente. Por lo tanto, la escalera que lleva a la equidad social y de género es todavía muy larga y no todos los sujetos, países y culturas, se encuentran en el mismo escaño.

Las preguntas de investigación que se hicieron al inicio de este trabajo se abordaron con el análisis histórico de las teorías feministas y de género y con la metateorización que abarcó a cinco de las teorías centrales del feminismo. Esta reflexión teórica abre la necesidad de estudiar las corrientes más actuales del feminismo como son el feminismo decolonial, el islámico, el feminismo factual o científico, el ciberfeminismo, el ecofeminismo entre otros, que con sus planteamientos teóricos y su praxis enriquecen la teorización feminista estudiando los problemas que hoy enfrentan hombres y mujeres.

Se puede observar en el continuo de las fases históricas por las que ha pasado el feminismo, que este va a continuar con mayor fuerza y aceptación en la comunidad científica pues sus argumentaciones están sustentadas con evidencia empírica y racionalidad, en tanto que la vertiente política de la lucha por el cambio va a llevar paulatinamente a mejorar las condiciones de las mujeres en todo el mundo. Hoy más que nunca es importante revisar las teorías feministas y aprender de lo que cada corriente aporta para la comprensión del mundo y su transformación. Con este breve escrito se espera haber contribuido en parte, a ese propósito.

\section{BIBLIOGRAFÍA}

Acker, J (1992) Gendering organizational theory, en Albert J. Mills and Peta Tancred (eds) Gendering organizational analysis. C.A: Sage, pp. 248-260.

Alcoff, L. (2002). Feminismo cultural vs Post-estructuralismo: la crisis de identidad de la teoría feminista, Revista Debats 76:18-41. Recuperado de http://148.202.18.157/sitios/catedrasnacionales/material/2010a/cristina_palomar/2.PDF

Banet-Weiser, S. (2018) Empowered: Popular feminism and popular misogyny. Duke: Duke University Press.

Barry, K. (2005). Teoría del feminismo radical: política de explotación sexual, en Amorós, C. y de Miguel, A. (eds.) Teoría feminista: de la Ilustración a la Globalización. Tomo I. De la Ilustración al segundo sexo. Madrid: Minerva ediciones, pp. 189-210.

Bengoechea B. M. (2003). El concepto de género en la sociolingüística, o cómo el paradigma de la dominación femenina ha malinterpretado la diferencia, en Silvia Tubert (coord.) Del sexo al género: los equívocos de un concepto. Madrid: Cátedra, pp 313-358.

Benhabib, S. (2005). Feminismo y posmodernidad: una difícil alianza, en Amorós, C. y de Miguel, A. (eds.), Teoría feminista: de la Ilustración a la Globalización Tomo I. De la Ilustración al segundo sexo. Madrid: Minerva Ediciones, pp. 319-342.

Benhabib, S. y Cornel, D. (eds) (1990) Teoría feminista y teoría crítica: ensayos sobre la política de género en las sociedades de capitalismo tardío. Valencia: Edicions Alfons El Magnanim.

Brown, R. M. (1974). The Last Straw. In Bunch and Myron (Eds) Class and feminism Baltimore: Diana Press, pp. 13-23.

Butler, J. (2004) Undoing Gender. New York and London: Routledge.

Butler, J. (2002) Críticamente subversiva, en R. M. Mérida (edit.) Sexualidades transgresoras. Una antología de estudios queer. Barcelona: Icaria, pp. 55-79. 
Cixous, H. (1991) The Laugh of the Medusa en R. R. Warhol/D. P. Herndl (eds.), Feminisms: An Anthology of Literary Theory and Criticism, New Brunswick, New Jersey: Rutgers University Press, pp. 334- 349.

Cobo, R. (2000). François Poullain de la Barre, sociológo y feminista en Sociología crítica y teoría feminista. Recuperado de https://masteres.ugr.es/gemma/pages/actividades/actividadesgranada/20072008/rosa_cobo/

Collins, P.H. (1998) "It's all in the family. Intersections of Gender, Race and Nation". Hypatia, 13(3): 62-82.

De Beauvoir, S. (2005) El segundo sexo. Madrid: Cátedra.

De Miguel, A. (2007). Feminismo de la diferencia y últimas tendencias. En Los feminismos a través de la historia. Recuperado de http://www.mujeresenred.net/spip.php?article1312

Declaración de sentimientos (2021) Recuperado de https://www.mujeresenred.net/spip.php?article2260

Firestone, S. (1976) La dialéctica del sexo. En defensa de la revolución feminista. Barcelona: Kairos. Flax, J. (1995) Psicoanálisis y feminismo. Pensamientos fragmentarios. España: Ediciones Cátedra. Foucault, M. (2005) Historia de la sexualidad. México: Siglo XXI editores.

Fraser, N. (1990) ¿Qué tiene de crítica la teoría crítica? Habermas y la cuestión del género, en Benhabib, S. y Cornell, D. : Teoría feminista y teoría crítica: ensayos sobre la política de género en las sociedades del capitalismo tardío, Valencia: Edicions Alfons el Magnànim, Institució Valenciana d'Estudis i Investigació, pp 119-149.

Fundación para el progreso (2021) Decálogo del feminismo liberal. Recuperado de https://fppchile.org/es/blog/decalogo-del-feminismo-liberal/

Haraway, D.J. (1989) Primate Visions: Gender, Race, and Nature in the World of Modern Science. Nueva York: Routledge.

Haraway, D.J. (1991) Ciencia, cyborgs y mujeres. Madrid: Cátedra.

Harding, S. (1996) Ciencia y feminismo. España: Ediciones Morata.

Hernández, V. (2003) “¿Podemos hablar de un feminismo posmoderno? Relación feminismoposmodernidad”. Graffylia. Revista de la Facultad de Filosofía y Letras de la BUAP, 1:53-66.

Hirschfeld, M. (2000) Homosexuality of Men and Women. New York: Prometheus.

Ibarra, E. y Montaño L. (comps.) (1990). Teoría de la Organización. Fundamentos y controversias. México: Universidad Autónoma Metropolitana- Iztapalapa.

Irigaray, L. (1982) Ese sexo que no es uno. Madrid: Saltes, D.L.

Irigaray, L. (2007) Speculum: Espéculo de la otra mujer. Madrid: Editorial Akal.

Jule, A. (2014) Gender Theory, en Michalos A.C. (eds) Encyclopedia of Quality of Life and WellBeing Research. Springer, Dordrecht. https://doi.org/10.1007/978-94-007-0753-5_1137

Kristeva, J. (1997) Al comienzo era el amor. Psicoanálisis y fe. Madrid: Gedisa.

Lauretis, Theresa de (1991) Feminist Studies / Critical Studies. USA: Indiana University Press

Lauretis, Theresa de (1992) Alicia ya no. Feminismo, semiótica, cine. España: Ediciones Cátedra.

Lerner, G. (1990) La creación del patriarcado. Barcelona: Editorial Crítica.

Madoo P. y Niebrugge-Brantley, J. (2004) Contemporary Feminist Theory, en George Ritzer y Douglas Goodman Modern sociological theory. New York: Mc Graw Hill, pp 456-480.

Mayobre Rodríguez, P. (2006). La formación de la identidad de género. Una mirada desde la filosofía en Esteve Zarazaga, J. M. y Vera Vila, J., Educación Social e Igualdad de Género. Málaga: Ayuntamiento de Málaga, pp. 21 a 59.

Mayobre, P. y Caruncho, C. (1998). Psicoanálisis, hermenéutica y género, en Agís Villaverde Marcelino Horizontes de la hermenéutica. Actas de los Encuentros Internacionales de Filosofía en el camino de Santiago Universidad de Santiago de Compostela, pp. 495-514. Consultado el 20 de mayo de 2020 de http://webs.uvigo.es/pmayobre/indicedearticulos.htm

McAffee, N. (2016). Feminist Political Philosophy. Stanford Encyclopedia of Philosophy. Recuperado de http://plato.stanford.edu/entries/feminism-class/

Mead, M. (2006) Sexo y temperamento. Barcelona: Paidos.

Millet, K. (1995) Política sexual. Madrid: Cátedra.

Moi, Toril (2001) What is a Woman? And Other Essays, España: Oxford University Press. 
Navarro, M. y Stimpson, C. (1998). Un Nuevo saber. Los estudios de mujeres. México: Fondo de Cultura Económica.

Poulain de la Barre, F. (1673). De l'égalité des deux sexes; discours physique et moral oú l'on volt l'importance de se défaire des préjugez,- Paris: chez Jean du Puis.

Puleo, A. (2021) Lo personal es político: el surgimiento del feminismo radical. Kate Millet. Recuperado de https://www.mujeresenred.net/spip.php?article2061

Toledo-Nickles, U. (2008) "El Esquema Metateórico de Ritzer desde la Metodología de los Programas de Investigación”. Cinta de Moebio, 33:204-218 http://dx.doi.org/10.4067/S0717554X2008000300004.

Tong, R. (2001) Feminist theory. International Encyclopedia of the Social \& Behavioral Sciences. Recuperado de https://www.sciencedirect.com/topics/social-sciences/feminist-theory

Tubert, S. (2009). Psicoanálisis, feminismo y posmodernismo. Recuperado de http://www.psiconet.com/foros/genero/posmo.htm

Tubert, S. (2003). La crisis del concepto de género, en S. Tubert (edit.) Del sexo al género: los equívocos de un concepto. Madrid: Cátedra, pp. 7-38.

Varela, N. (2005) Feminismo para principiantes. Barcelona: Ediciones B. Walby, S.

Wollstonecraft, M. (2014) Vindicación de los derechos de la mujer. Madrid: Akal.

Wilchins, R. (2004). Queer Theory, Gender Theory: An Instant Primer. Los Ángeles: Alyson Books.

\section{Breve currículo}

\section{Dra. Maricela Guzmán Cáceres}

Profesora Investigadora en la Escuela de Ciencias de la Comunidad de la Universidad Autónoma de Coahuila. Miembro del Sistema Nacional de Investigadores. Socióloga, Maestra en Investigación Educativa y Maestra en Ciencias Sociales. Realizó un posdoctorado en el Centro de Investigaciones Interdisciplinarias en Ciencias y Humanidades de la UNAM. Ha sido directora y colaboradora en siete proyectos de investigación financiados por Conacyt. Su trabajo de investigación está publicado en un libro, capítulos de libro, artículos y divulgado en Congresos en México y el extranjero. Líneas de investigación: "Teoría de género" y "Sociología de las Organizaciones", "Liderazgo y Desarrollo Humano". 